\title{
Single-Phase CT Aligned to Gated PET for Respiratory Motion Correction in Cardiac PET/CT
}

\author{
R. Glenn Wells ${ }^{1}$, Terrence D. Ruddy ${ }^{1,2}$, Rob A. DeKemp ${ }^{1,2}$, Jean N. DaSilva ${ }^{1}$, and Rob S. Beanlands ${ }^{1,2}$ \\ ${ }^{1}$ Division of Cardiology, Department of Medicine, University of Ottawa Heart Institute, Ottawa, Ontario, Canada; and ${ }^{2}$ Divsion of \\ Nuclear Medicine, Department of Medicine, University of Ottawa Heart Institute, Ottawa, Ontario, Canada
}

Respiratory motion can induce artifacts in cardiac PET/CT because of the misregistration of the CT attenuation map and emission data. Some solutions to the respiratory motion problem use 4-dimensional CT, but this increases patient radiation exposure. Realignment of 3-dimensional CT and PET images can remove apparent uptake defects caused by mispositioning of the PET emission data into the lung regions on the CT scan. This realignment is typically done as part of regular clinical quality assurance. We evaluated a method to improve on this standard approach, without increasing the radiation exposure to the patient, by acquiring a respiration-gated PET scan and separately aligning the 3-dimensional CT scan to each phase of the PET study. Methods: Three hundred ten clinical PET perfusion scans ( ${ }^{82} \mathrm{Rb}[n=187]$ and ${ }^{13} \mathrm{~N}$-ammonia [ $\left.n=123\right]$ ) were retrospectively assessed. Studies were respiration-gated, and motion was measured between inspiration and expiration phases. Those studies with motion $\geq 8 \mathrm{~mm}$ were evaluated for significant differences between inspiration and expiration. Studies with significant differences were reprocessed with the phase-alignment approach. The observed motion with ${ }^{82} \mathrm{Rb}$ and ${ }^{13} \mathrm{~N}$-ammonia for rest and stress imaging was also compared. Results: Twentythree scans $(7.41 \%)$ had motion $\geq 8 \mathrm{~mm}$, and 9 of these had significant differences between inspiration and expiration, suggesting the presence of respiratory artifacts. Phase-aligned respiratory motion compensation reduced this difference in 8 of 9 cases (89\%). No significant differences were observed between ${ }^{82} \mathrm{Rb}$ and ${ }^{13} \mathrm{~N}$-ammonia, and motion during stress imaging was correlated with motion at rest $(r=0.61, P<0.001)$. Conclusion: Phase-aligned correction improves the consistency of PET/CT perfusion images by reducing discrepancies caused by respiratory motion. This new approach to CT-based attenuation correction has no additional patient radiation exposure and may improve the specificity of PET perfusion imaging.

Key Words: cardiac PET/CT; respiratory motion; CT attenuation correction; ammonia; ${ }^{82} \mathrm{Rb}$

J Nucl Med 2010; 51:1182-1190

DOI: 10.2967/jnumed.109.070011

Received Aug. 28, 2009; revision accepted Mar. 19, 2010.

For correspondence or reprints contact: R. Glenn Wells, University of Ottawa Heart Institute, Cardiac Imaging, 1st Floor, Rm. H-1118, 40

Ruskin St., Ottawa, ON, Canada, K1Y 4W7.

E-mail: gwells@ottawaheart.ca

COPYRIGHT $\odot 2010$ by the Society of Nuclear Medicine, Inc.
A ccurate attenuation correction (AC) is a major clinical advantage of PET, compared with SPECT, and has been well accepted when the attenuation map is formed using data from radioactive transmission sources. However, respiratory motion can induce artifacts in cardiac PET/CT because of misregistration of the $\mathrm{CT}$ attenuation map and emission data. Misregistration has been shown to occur in up to $40 \%$ of clinical cardiac PET/CT studies (1). The most common artifact is an apparent decrease in uptake in the anterior or lateral wall due to a mispositioning of the activity from the heart into the low-attenuating tissues of the lungs. Many studies have examined the effect of this misalignment and found that motion of $6-8 \mathrm{~mm}$ produces mild to moderate errors, and motion $>8 \mathrm{~mm}$ leads to severe errors that may affect clinical interpretation $(1,2)$. Several approaches have been suggested to reduce the impact of this problem including simple realignment of the CT scan and the PET data (2,3), slow CT scans (4), and methods that estimate the motion from respiration-gated PET emission data and use these motion fields to transform a static CT scan and align it with the PET data $(5,6)$. Other approaches to the respiratory motion problem use a 4-dimensional CT scan (4D-CT) - that is, an acquisition of multiple CT volumes over the respiratory cycle. These latter techniques include the use of maximum-intensity CT images (7), respiration-averaged CT (1,7-9), complete phase-matched 4-dimensional AC (9-11), and 4-dimensional reconstruction with motion estimates based on the 4D-CT data (12). A drawback of the 4D-CT scan is the increase in patient dose over that from a standard helical 3-dimensional (3D) CT scan. The effective dose for a 4D-CT cardiac scan is estimated at $1.4 \mathrm{mSv}(1,13)$, whereas that for a cardiac attenuation-map helical CT scan is typically about $0.5 \mathrm{mSv}$ and can be as low as $0.15 \mathrm{mSv}$ (4). Obtaining a 4D-CT scan at both rest and stress for cardiac perfusion imaging would result in an increase in effective dose of $\geq 2 \mathrm{mSv}$. Because the dose for the emission component of an ${ }^{13} \mathrm{~N}$-ammonia $\left(\mathrm{NH}_{3}\right)$ rest-stress perfusion study is approximately $2.2 \mathrm{mSv}$ (14), the dose from the CT portion of the study represents a significant fraction of the total effective dose. Minimizing 
total radiation dose is becoming more important with increasing availability of competing technologies and greater public concern over the potential long-term adverse effects of the cumulative radiation dose from multiple imaging studies.

Khurshid et al. (3) showed that realignment of the heart in the CT and PET images can remove apparent uptake defects caused by mispositioning of the PET emission data into the lung regions on the CT scan. In their study of 50 patients with visually apparent hypoperfusion of the myocardium (3), Khurshid et al. eliminated the defect in $44 \%$ of the cases. This technique compensates for the change in the mean position of the heart in the CT and PET datasets but does not accurately correct that portion of the PET data for which respiratory motion takes the heart away from the mean position. An improvement to this technique may, therefore, be achieved by acquiring a respiration-gated PET scan and separately aligning the CT image to the PET image at each phase of the breathing cycle. The objective of this study was to determine the extent to which manual alignment of a single-phase CT scan with each phase of a respiration-gated PET emission scan will reduce the artifacts caused by respiratory motion in clinical cardiac ${ }^{82} \mathrm{Rb}$ and $\mathrm{NH}_{3}$ PET/CT studies. A secondary objective was to compare the motion seen in ${ }^{82} \mathrm{Rb}$ and $\mathrm{NH}_{3}$ studies during rest and stress imaging.

\section{MATERIALS AND METHODS}

\section{Image Acquisition}

Images were retrospectively examined from 310 scans drawn from the studies of 190 patients. The dataset consisted of ${ }^{82} \mathrm{Rb}$ cardiac PET scans ( $n=187 ; 103$ rest and 84 stress) obtained from 113 patients between December 2007 and March 2008 and 123 $\mathrm{NH}_{3}$ scans (53 stress and 70 rest) acquired from 77 patients between December 2007 and November 2008. These studies represented all of the scans for which a respiration-gating signal was successfully obtained during the indicated periods, and the resulting images were of sufficient quality to permit measurement of motion. Respiration-gated data were not available in some patients, for several reasons. Operator error led to the loss of a large percentage of potential datasets. Of those cases for which it was noted that respiration-gating was not possible, the causes included loss of visualization of the reflector block because of patient size or voluntary motion ( $\sim 7 \%$ of patients) or the use of a warming blanket ( $\sim 6 \%$ of patients). A respiratory signal was acquired but resulted in poor gated image quality because of low total counts in $3 \%$ of $\mathrm{NH}_{3}$ scans and $6 \%$ of ${ }^{82} \mathrm{Rb}$ scans (total, 17 scans). In addition, unsuccessful gating due to shallow or erratic breathing leading to the loss of a large fraction of the acquired counts and resulting poor image quality occurred in an additional twelve ${ }^{82} \mathrm{Rb}$ scans $(6 \%)$. The demographics of the patients included in the study are described in Table 1.

All studies were performed according to standard clinical imaging protocols in accordance with the American Society of Nuclear Cardiology guidelines (15). The average injected doses were $16.9 \pm 4.7 \mathrm{MBq} / \mathrm{kg}$ for ${ }^{82} \mathrm{Rb}$ and $5.4 \pm 1.1 \mathrm{MBq} / \mathrm{kg}$ for $\mathrm{NH}_{3}$. Acquisition was started immediately after tracer injection, and the study lasted $10 \mathrm{~min}$ for ${ }^{82} \mathrm{Rb}$ and $20 \mathrm{~min}$ for $\mathrm{NH}_{3}$. Data were acquired in 2 dimensions with list mode on a Discovery Rx PET/ CT scanner (GE Healthcare) equipped with a 64-slice CT scanner. For this study, PET data starting from $2.5 \mathrm{~min}$ after injection $\left({ }^{82} \mathrm{Rb}\right)$ or 5 min after injection $\left(\mathrm{NH}_{3}\right)$ were reprocessed and binned into 8 respiratory phases using an acceptance window of 15 breaths per minute $\pm 50 \%$. The respiratory trigger was obtained using a Real-Time Patient Management device (Varian Medical Systems), which optically tracks the transaxial motion of the chest using external markers. For AC, a single fast CT acquisition (1.5 s) was obtained at mid- to end-expiration immediately before (rest studies) or after (stress studies) the PET acquisition. Patients were instructed to maintain normal shallow breathing throughout the PET and CT acquisitions.

All studies were reconstructed using filtered backprojection, followed by postfiltering with a $14-\mathrm{mm}\left(\mathrm{NH}_{3}\right)$ or $16-\mathrm{mm}\left({ }^{82} \mathrm{Rb}\right)$ 2-dimensional Hann filter as per our clinical imaging protocol. The CT image used for AC was aligned with a static (ungated) PET image using vendor-supplied quality-assurance software that allows manual rigid-body registration. The respiration-gated images were then exported offline and processed using custom in-house software.

\section{Respiratory-Phase Image Registration}

Each phase of the respiration-gated PET image set was registered to the first phase (typically near end-expiration).

\begin{tabular}{|c|c|c|c|}
\hline Demographic & $\mathrm{NH}_{3}{ }^{*}$ & ${ }^{82} \mathrm{Rb}^{\dagger}$ & Combined \\
\hline No. of patients & 77 & 113 & 190 \\
\hline Age $(y)$ & $63 \pm{ }^{*} 9$ & $62 \pm 12$ & $62 \pm 11$ \\
\hline No. men & $56(73 \%)$ & $71(63 \%)$ & $127(67 \%)$ \\
\hline Mean body mass index $\pm S D$ & $30.4 \pm 6.3$ & $29.8 \pm 6.8$ & $30.0 \pm 6.6$ \\
\hline Smoking (\%) & 61 & 68 & 65 \\
\hline Family history (\%) & 47 & 52 & 50 \\
\hline DM (\%) & 28 & 25 & 26 \\
\hline HTx (\%) & 63 & 73 & 69 \\
\hline HLipid (\%) & 67 & 69 & 68 \\
\hline
\end{tabular}


Registration was achieved by translating the images in the 3 Cartesian directions, using trilinear interpolation, and minimizing the pixelwise mean squared difference between the phase images in the region of the heart. The region of the heart was a rectangular volume enclosing the heart from the static PET image, ensuring that the heart would not move out of the volume during respiration. The default volume was adjusted to exclude strong extracardiac sources such as the stomach and liver. A 3D gridsearch technique was used, with a search radius of $15 \mathrm{~mm}$ to optimize the registration. The maximum motion in each direction and the maximum displacement between any 2 respiratory phases were determined from the registration transformations. The displacement corresponds to the magnitude of the motion vector defined by the motion in the 3 Cartesian directions. Motion for ${ }^{82} \mathrm{Rb}$ and $\mathrm{NH}_{3}$ studies was compared, as was the motion in rest and stress scans. The accuracy of the registration was assessed by manually shifting the first respiratory-phase image of each study by a fixed amount $-4 \mathrm{~mm}$ in the $x$ and $y$ directions and $8 \mathrm{~mm}$ in the $z$ (axial) direction-and repeating the registration with the original image. Repeatability was assessed by repeating the registration using the second respiratory phase as the reference phase instead of the first. The mean and SD of the differences in maximum motion were measured.

\section{The No-Motion Database}

To evaluate the variation in the distribution of activity over the respiratory cycle, the 2 gates of the study nearest end-inspiration ( $25 \%$ of the data) were summed, as were the 2 gates nearest end-expiration, creating inspiration and expiration images. The images were summed to reduce the noise levels in the gated ${ }^{82} \mathrm{Rb}$ images and to increase the proportion of the image data being evaluated as the contribution of any differences found would be diluted when summed into the static PET image. Compressing the number of bins from 8 to 4 is also more consistent with the optimal number of $5-6$ bins reported by others $(16,17)$. The $\mathrm{NH}_{3}$ images were similarly processed for consistency. Other factors such as noise in the images and the changing shape of the heart with respiration (18) might also influence the apparent activity distribution at each phase. Therefore, a database was created to estimate this variability using images with displacement $<6 \mathrm{~mm}$ during the respiratory cycle. A cutoff of $6 \mathrm{~mm}$ was chosen because others have suggested that movement $<6 \mathrm{~mm}$ does not cause clinically significant errors, and it has been used to distinguish mild from moderate and severe motion $(1,2)$. To create the no-motion database, the difference between the inspiration and expiration images was analyzed with 4DM software (INVIA Medical Imaging Solutions) using polar maps divided according to the standard 17-segment heart model. Polar maps were normalized to the maximum value and average segment scores expressed as a percentage of the maximum. SDs for the difference between inspiration and expiration were estimated for each of the 17 segments. Separate databases were created for each tracer.

\section{Phase-Aligned Correction of Images with Displacement $\geq 8 \mathrm{~mm}$}

Those studies with respiratory displacement $\geq 8 \mathrm{~mm}$ were then selected for further evaluation and potential reprocessing using the proposed phase-alignment method. These studies represent those most likely to contain residual respiratory artifacts after alignment of the CT image to the mean (ungated) PET position. In addition, the studies with the largest motion are those most likely to contain morphologic changes in the distribution of tissues. Variation in the relative position of the liver with respect to the heart is known to cause artifacts in the PET image $(1,19)$, and this source of error would not be corrected by the phase-alignment approach. Assessment of the phase-alignment correction technique on the subset of cases with largest displacement thus represents a lower limit in performance, and application of the method to images with less motion is expected to result in equal or improved results.

The images with displacement $\geq 8 \mathrm{~mm}$ were compared with the no-motion database to determine which of these showed significant variation in the apparent activity during respiration. The difference between inspiration and expiration was considered significant if the difference in a single segment was greater than 3.0 SDs away from the database mean value for that segment or if the differences in any 2 adjacent segments were both greater than 2.2 SDs away from the database mean value for that segment. These criteria give an $\alpha$-value of less than 0.05 , using a Sidák correction for multiple comparisons.

Scans with significant differences between inspiration and expiration were then reprocessed using phase-aligned CT AC. The CT scan was manually aligned with the PET phase corresponding to end-inspiration, a new gated PET image was reconstructed, and the end-inspiration PET phase was extracted (Supplemental Fig. 1). Similarly, the CT scan was aligned with the end-expiration phase to create a CT expiration PET image, and the expiration phase extracted. With this approach, a phase-aligned PET image set was created. The images from the phase-aligned reconstructions were compared with the original reconstructions (with CT images aligned to the ungated PET image) to determine the extent to which errors associated with respiration could be resolved by manual alignment of the CT scan.

As the degree of motion decreases, the contribution of respiratory motion to any differences seen between inspiration and expiration may also diminish. Therefore, it is also of interest to evaluate how the phase-alignment approach performs on those cases with a lesser degree of motion. With this in mind, we briefly evaluated the effect of lowering our cutoff from 8 to $7 \mathrm{~mm}$ and considered those cases with a measured displacement between 7 and $8 \mathrm{~mm}$.

\section{RESULTS}

\section{Evaluation of Motion}

A summary of the measured motion in the scans is given in Table 2. Shown are the 3 Cartesian directions and the maximum displacement between any 2 respiratory phases of a scan. The maximum displacements are displayed separately for rest and stress for each tracer in Figure 1. For both ${ }^{82} \mathrm{Rb}$ and $\mathrm{NH}_{3}$, the greatest amount of motion was in the axial (cranial-caudal) direction, as is expected with respiratory motion. Only 2 scans, both stress ${ }^{82} \mathrm{Rb}$, had a transaxial motion $>6 \mathrm{~mm}$. In the axial direction, thirty-five ${ }^{82} \mathrm{Rb}$ scans $(19 \%)$ and $29 \mathrm{NH}_{3}$ scans $(24 \%)$ had motion $>$ $6 \mathrm{~mm}$, and twelve ${ }^{82} \mathrm{Rb}$ scans $(6 \%)$ and $4 \mathrm{NH}_{3}$ scans $(3 \%)$ had motion $\geq 8 \mathrm{~mm}$. Fifty-four ${ }^{82} \mathrm{Rb}$ studies $(29 \%)$ and 42 $\mathrm{NH}_{3}$ scans $(34 \%)$ had displacement $>6 \mathrm{~mm}$, and fourteen ${ }^{82} \mathrm{Rb}$ studies $(7.5 \%)$ and $9 \mathrm{NH}_{3}$ scans $(7.3 \%)$ had displacement $\geq 8 \mathrm{~mm}$.

Realigning the first phase of each scan after a known shift of $4 \mathrm{~mm}$ in both transaxial directions and $8 \mathrm{~mm}$ in the 
TABLE 2. Measured Patient Motion

\begin{tabular}{|c|c|c|c|c|c|}
\hline Radiotracer & & Left-right (mm) & Anterior-posterior (mm) & Cranial-caudal (mm) & Displacement (mm) \\
\hline \multirow[t]{6}{*}{$\mathrm{NH}_{3}$} & Mean & 2.5 & 1.7 & 4.9 & 5.4 \\
\hline & SD & 0.9 & 0.8 & 1.8 & 1.7 \\
\hline & Maximum & 5.7 & 4.1 & 11.0 & 11.4 \\
\hline & Minimum & 0.8 & 0.6 & 2.0 & 2.2 \\
\hline & $>6 \mathrm{~mm}$ & 0 & 0 & 29 & 42 \\
\hline & $\geq 8 \mathrm{~mm}$ & 0 & 0 & 4 & 9 \\
\hline \multirow[t]{6}{*}{${ }^{82} \mathrm{Rb}$} & Mean & 2.9 & 2.3 & 4.4 & 5.4 \\
\hline & SD & 1.0 & 0.9 & 2.0 & 2.0 \\
\hline & Maximum & 8.5 & 7.1 & 14.3 & 15.1 \\
\hline & Minimum & 1.1 & 0.8 & 1.3 & 2.1 \\
\hline & $>6 \mathrm{~mm}$ & 2 & 1 & 35 & 54 \\
\hline & $\geq 8 \mathrm{~mm}$ & 1 & 0 & 12 & 14 \\
\hline
\end{tabular}

axial direction produced a mean error in the transaxial $(x$ and $y$ ) and axial $(z)$ directions of $0.14,0.12$, and $-0.03 \mathrm{~mm}$, respectively, with an SD of $0.04,0.03$, and $0.02 \mathrm{~mm}$, respectively, for ${ }^{82} \mathrm{Rb}$. For $\mathrm{NH}_{3}$, the results were a mean error of $0.16,0.12$, and $-0.02 \mathrm{~mm}$, respectively, with an $\mathrm{SD}$ of $0.06,0.04$, and $0.02 \mathrm{~mm}$, respectively. Repeating the registration using the second phase as the reference instead of the first produced differences for ${ }^{82} \mathrm{Rb}$, with a mean of $-0.02,-0.09$, and $-0.13 \mathrm{~mm}$, respectively, and an SD of $0.76,0.66$, and $0.84 \mathrm{~mm}$, respectively. For $\mathrm{NH}_{3}$, the differences in the registrations had a mean of $-0.08,-0.07$, and $-0.01 \mathrm{~mm}$, respectively, and an SD of $0.66,0.43$, and $0.50 \mathrm{~mm}$. Registration of an 8-phase study required $80 \mathrm{~s}$ on a $2.7-\mathrm{GHz}$ Intel Xeon single-core processor.

\section{Motion at Rest, Compared with During Stress}

Considering the subset of 74 paired ${ }^{82} \mathrm{Rb}$ rest-stress scans (total, 148 scans), the axial motion during stress imaging was not significantly greater than that at rest (mean, 4.6 vs. $4.3 \mathrm{~mm} ; P=0.08$; paired $t$ test). However,

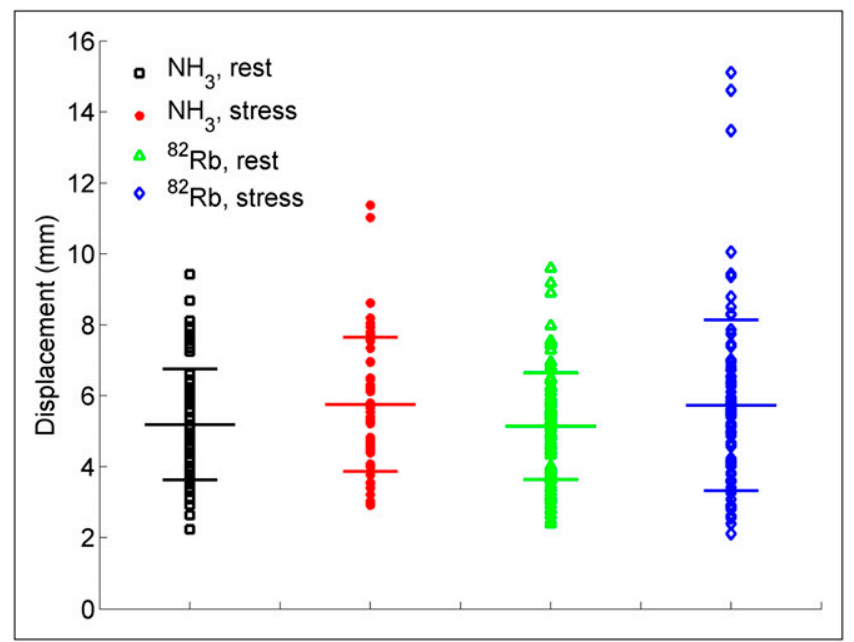

FIGURE 1. Displacement between inspiration and expiration of heart images acquired at rest and at stress using $\mathrm{NH}_{3}$ and ${ }^{82} \mathrm{Rb}$. Mean and $\mathrm{SD}$ for each distribution is shown with horizontal bars. of those paired scans with axial motion $\geq 8 \mathrm{~mm}, 3$ were at rest and 7 were at stress. If we consider displacement, then 4 resting studies had displacement $\geq 8 \mathrm{~mm}$, versus 7 at stress. With the 46 paired $\mathrm{NH}_{3}$ rest-stress scans (92 scans), stress imaging likewise did not produce significantly greater axial motion than rest imaging $(5.1$ vs. $4.7 \mathrm{~mm}$; $P=0.2$; paired $t$ test) and there was a similar percentage of studies with significant motion. Of those scans with axial motion $\geq 8 \mathrm{~mm}, 2$ were at rest and 2 were at stress. Of the scans with displacement $\geq 8 \mathrm{~mm}, 2$ were at rest and 4 were at stress.

Comparing axial motion for $\mathrm{NH}_{3}$ with the ${ }^{82} \mathrm{Rb}$ data, the average magnitude was not significantly different at rest $(P=0.14)$ or stress $(P=0.19)$, nor were the variances at rest $(P=0.37)$ or stress $(P=0.25)$; also, the correlation between rest and stress was not significantly different $(P=$ $0.11)$. Therefore, we also evaluated the combination of all ${ }^{82} \mathrm{Rb}$ and $\mathrm{NH}_{3}$ rest and stress studies. For the combined data, the stress motion averaged $4.8 \pm 2.1 \mathrm{~mm}$ and was significantly different $(P=0.03)$ from the rest motion $(4.5 \pm 1.7 \mathrm{~mm})$ (Fig. 1). Stress axial motion was correlated with rest with a Pearson correlation of $r=0.61(P<0.001)$ (Fig. 2).

Given the relationship between axial motion at rest and stress, we then also examined whether rest motion could be used to predict stress motion $>6 \mathrm{~mm}$. A receiveroperating-characteristic analysis on the combined reststress data produced an area under the curve of 0.85 , with a maximum accuracy of $85 \%$ (sensitivity, $77 \%$; specificity, $86 \%$ ) with a rest motion of $5.5 \mathrm{~mm}$ (Fig. 3). A negative predictive value of $96 \%$ was obtained at a cutoff of $4.0 \mathrm{~mm}$ of resting motion ( $45 \%$ of studies had resting axial motion $\leq$ $4 \mathrm{~mm}$ ). The positive predictive value was $80 \%$ at $7.0 \mathrm{~mm}$ and $100 \%$ at $7.4 \mathrm{~mm}$.

\section{Evaluation of Significant Respiratory Errors and Phase-Aligned Correction of Images with Motion $\geq 8 \mathrm{~mm}$}

The no-motion-difference polar maps had a mean bias $( \pm \mathrm{SD})$ in the 17 segments of $-0.14 \% \pm 1.43 \%$ for ${ }^{82} \mathrm{Rb}$ and $-0.84 \% \pm 1.45 \%$ for $\mathrm{NH}_{3}$. The segmental $\mathrm{SD}$ was 


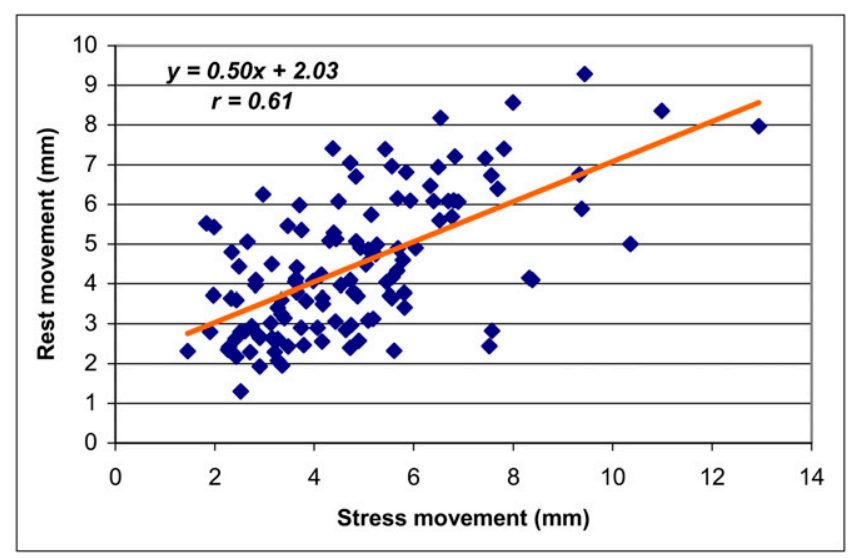

FIGURE 2. Correlation between all paired rest-stress studies_-both $\mathrm{NH}_{3}$ and ${ }^{82} \mathrm{Rb}(r=0.61, P<0.001)$.

$4.2 \% \pm 0.5 \%$ for $\mathrm{NH}_{3}$ and $5.6 \% \pm 0.5 \%$ for ${ }^{82} \mathrm{Rb}$. Our criteria of significant difference thus correspond to a difference in tracer uptake of $13 \%$ and $17 \%$ (3 SDs) in a single segment or $9.2 \%$ and $12 \%$ (2.2 SDs) in 2 adjacent segments for $\mathrm{NH}_{3}$ and ${ }^{82} \mathrm{Rb}$, respectively. Of the 23 images for which the displacement was measured as $\geq 8 \mathrm{~mm}, 9$ images showed significant differences between inspiration and expiration. These 9 images had displacements ranging from 8.0 to $14.6 \mathrm{~mm}$. Application of phase-aligned CT AC reduced the number of segments with differences $\geq 2.2$ SDs in 8 of these cases. Phase alignment removed all significant differences in 4 cases. In 1 case, there was no change in the number of significantly different segments. The number of significantly different segments for the 9 scans before and after phase-aligned correction is given in Table 3.

Decreasing our cutoff for consideration from 8 to $7 \mathrm{~mm}$ resulted in an additional 40 cases, of which 6 had significant

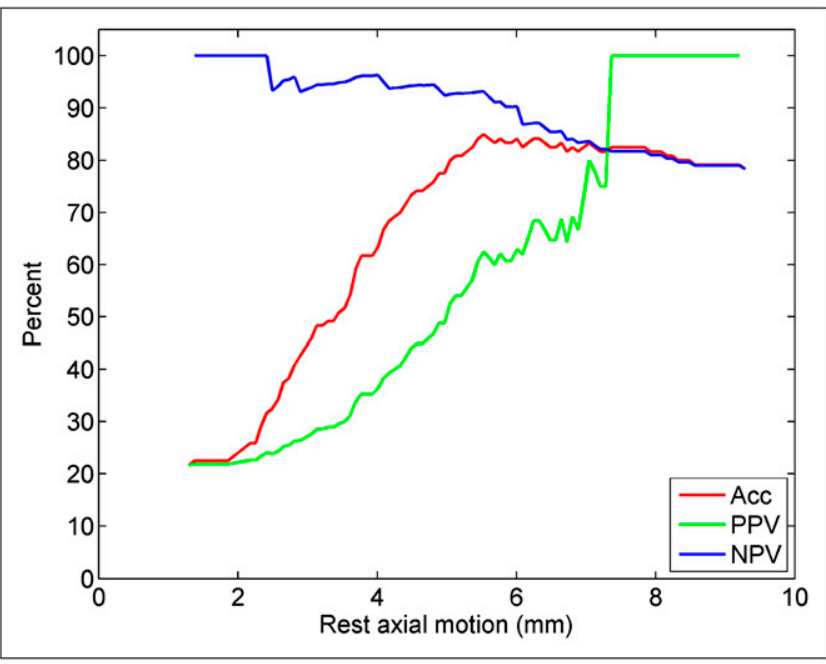

FIGURE 3. Positive predictive value (PPV), negative predictive value (NPV), and accuracy (ACC) of resting axial motion for predicting axial motion of $>6 \mathrm{~mm}$ at stress. differences between inspiration and expiration. Phase-aligned correction removed these differences in 2 cases, decreased the differences in 3 cases, and afforded no improvement in 1 case. These scans are also included at the bottom of Table 3 .

The image in Figure 4 demonstrates phase-alignment resolution of all significant differences between inspiration and expiration (scan 2 from Table 3). Summation of the respiratory phases after phase-aligned CT correction produces an image that, compared with the original static PET image, has segmental differences of up to 5\% (Fig. 5).

\section{DISCUSSION}

In this study, $41 \%$ of images acquired at rest and stress using $\mathrm{NH}_{3}$ and ${ }^{82} \mathrm{Rb}$ had respiratory motion $>6 \mathrm{~mm}$, and $7.5 \%$ had motion $\geq 8 \mathrm{~mm}$; respiratory motion at stress was significantly correlated with motion measured at rest; and $39 \%$ of those studies with motion $\geq 8 \mathrm{~mm}$ had significant differences in apparent tracer uptake between inspiration and expiration and $89 \%$ of these differences were reduced with phase-aligned CT AC.

\section{Motion Artifacts and Phase-Aligned Correction}

By obtaining a respiration-gated PET reconstruction, it is possible to assess the images for the presence of respiration-induced artifacts by comparing the images acquired near inspiration with those acquired near expiration. In those cases for which significant differences exist, the PET images could be reprocessed with a phase-aligned CT correction. Applying the phase-aligned correction would entail additional quality-assurance steps to align the CT scan separately to each phase but would be required only in the small percentage of cases that showed evidence of respiration-induced changes. The resultant respirationgated PET image set could then be summed to provide an image equivalent to the static image currently used in clinical evaluation. Alternatively, the gated images could be registered and aligned to correct for motion before summation, reducing motion blurring and potentially further improving the final image quality, as has been suggested by others $(20,21)$.

The average respiratory motion in this study was $4.6 \mathrm{~mm}$ axially and less than $3 \mathrm{~mm}$ transaxially. The magnitude of motion was less than has been reported in some studies $(22,23)$, though consistent with the values reported in others (17). This may simply be due to the measurement technique. Because measurement was taken from the gated PET study, the use of 8 respiratory gates limits the temporal resolution, and rapid motion-for example, at the start of inspiration-may not be adequately captured. The apparent motion would consequently be reduced. In addition, the respiratory gating was phase-based. Other groups have suggested that amplitude-based respiratory gating may more accurately represent the true extent of the motion (17). Dawood et al. (17) showed a 2-fold reduction in respiratory amplitude with phase-based gating, compared with amplitude-based gating. Unfortunately, many of the 
TABLE 3. Number of Segments That Are Significantly Different Between Inspiration and Expiration Before and After Phase-Aligned CT Correction

\begin{tabular}{|c|c|c|c|c|c|}
\hline \multirow[b]{3}{*}{ Scan } & \multirow{3}{*}{$\begin{array}{c}\text { Maximum } \\
\text { displacement } \\
\text { measured }(\mathrm{mm})\end{array}$} & \multicolumn{4}{|c|}{ Segments with... } \\
\hline & & \multicolumn{2}{|c|}{$\begin{array}{l}\text { CT aligned to } \\
\text { static PET }\end{array}$} & \multicolumn{2}{|c|}{$\begin{array}{l}\text { CT phase-aligned } \\
\text { to gated PET }\end{array}$} \\
\hline & & $\geq 3 \mathrm{SDs}$ & $\geq 2.2 \mathrm{SDs}$ & $\geq 3 \mathrm{SDs}$ & $\geq 2.2 \mathrm{SDs}$ \\
\hline 1, $\mathrm{NH}_{3}$ rest & 8.0 & 0 & 6 & 0 & 3 \\
\hline $2, \mathrm{NH}_{3}$ rest & 8.7 & 3 & 4 & 0 & 0 \\
\hline $3, \mathrm{NH}_{3}$ stress & 11.4 & 4 & 5 & 0 & 3 \\
\hline $4, \mathrm{NH}_{3}$ stress & 8.6 & 0 & 3 & 1 & 3 \\
\hline $5,{ }^{82} \mathrm{Rb}$ rest & 8.0 & 1 & 4 & 0 & 0 \\
\hline $6,{ }^{82} \mathrm{Rb}$ stress & 14.6 & 0 & 2 & 0 & 0 \\
\hline $7,{ }^{82} \mathrm{Rb}$ stress & 13.5 & 1 & 3 & 0 & 2 \\
\hline $8,{ }^{82} \mathrm{Rb}$ stress & 9.4 & 1 & 10 & 1 & 4 \\
\hline $9,{ }^{82} \mathrm{Rb}$ stress & 8.3 & 1 & 5 & 0 & 1 \\
\hline $10, \mathrm{NH}_{3}$ rest & 7.7 & 4 & 7 & 0 & 2 \\
\hline $11, \mathrm{NH}_{3}$ rest & 7.4 & 2 & 3 & 1 & 2 \\
\hline $12, \mathrm{NH}_{3}$ rest & 7.7 & 0 & 4 & 0 & 4 \\
\hline $13, \mathrm{NH}_{3}$ stress & 7.6 & 1 & 2 & 1 & 1 \\
\hline $14,{ }^{82} \mathrm{Rb}$ stress & 7.4 & 0 & 2 & 0 & 0 \\
\hline $15,{ }^{82} \mathrm{Rb}$ stress & 7.5 & 1 & 5 & 0 & 1 \\
\hline \multicolumn{6}{|c|}{$\begin{array}{l}\text { Reduction is significant for both } \geq 3 \text {-SD segments (1-tailed paired } t \text { test, } P<0.05 \text { ) and } \geq 2.2 \text {-SD segments }(1 \text {-tailed paired } t \text { test } \\
P<0.01 \text { ). }\end{array}$} \\
\hline
\end{tabular}

clinical systems currently available do not provide for amplitude-based gating, including the Discovery Rx system used in this study. Amplitude-based gating might lead to a segmentation of the PET scan that is more consistent with a well-defined fixed CT position and thus might further improve the accuracy of this approach.

Only 1 of the 9 cases that had evidence of respirationrelated artifacts showed no improvement with the phasealignment approach. This is likely due to morphologic changes in the CT image between inspiration and expiration as described by Gould et al. (1). McQuaid and Hutton also showed that, although misregistration of the heart produced the largest errors in PET images (84\%), misalignment of the liver and lungs could also produce errors
(59\% and 28\%, respectively) (19). These changes in the relative location of the liver and lungs with respect to the heart during respiration prevent rigid-body registration from providing an accurate alignment of the expiration CT map to all of the other phases. If a 4D-CT-based AC removed the differences seen in this 1 case, it would confirm morphologic changes as the source of the artifact, but 4D-CT was not available for this retrospective study. A possible alternative solution is to use nonrigid registration of the CT image to the different respiratory phases guided by the gated PET images, such as has been proposed by others $(5,12,24)$. Nonrigid registration would address this limitation of the phase-alignment technique, without the increase in patient radiation dose and image acquisition
A
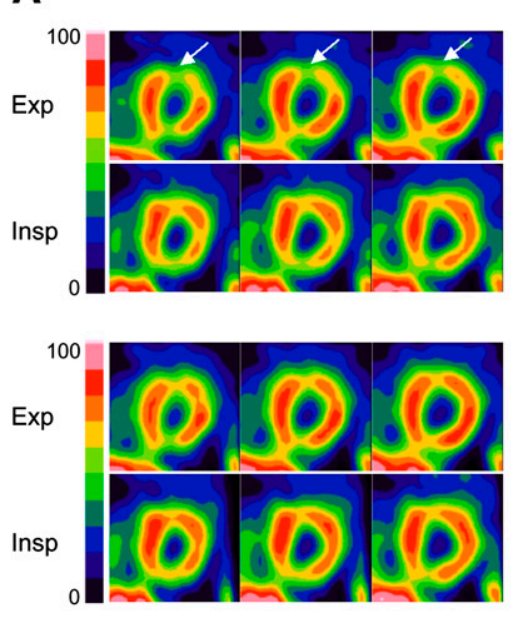

B
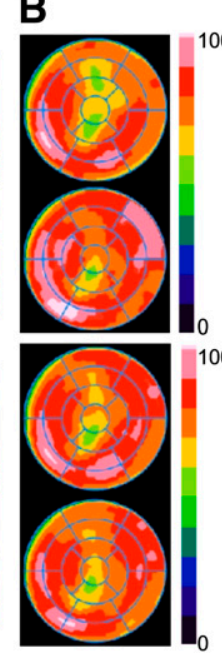

C

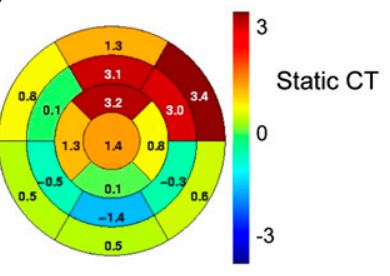

100

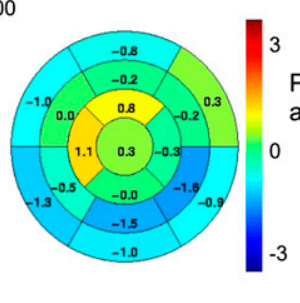

FIGURE 4. Image demonstrating that phase alignment removes differences between inspiration (insp) and expiration (exp). Case shown is for $\mathrm{NH}_{3}$ study with displacement of $8.7 \mathrm{~mm}$; all images acquired at rest. Top set of images is corrected with static CT image that was aligned to ungated PET images. Bottom images are those corresponding to a phase-aligned correction. Short-axis slices are shown on left (column A), with corresponding polar map representations (column B). Column C shows polar map comparison to no-motion database in which values correspond to number of $\mathrm{SDs}$ of difference (insp-exp). Change in agreement of anterior wall is indicated by arrows. Phase-aligned images show no significant differences. 
FIGURE 5. Example of difference in ungated PET image (top), compared with sum of respiratory phases for phase-aligned correction (bottom). Short-axis slices are shown in column A, with corresponding polar maps in column B. The 2 polar maps (C) show up to $5 \%$ segmental difference.

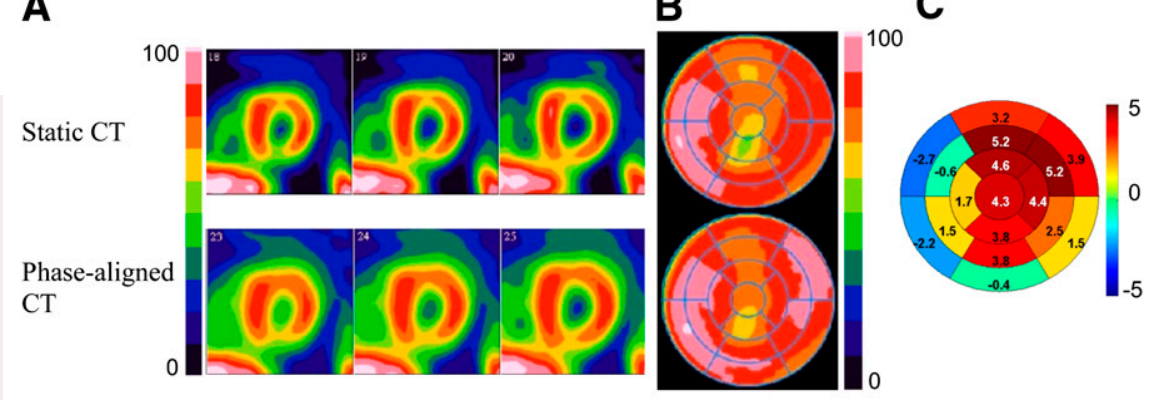

complexity that is associated with 4D-CT acquisitions and should further reduce the differences caused by respiratory motion. Another potential solution is to use the no-motion-difference database to identify those phases of the image that are inconsistent with the end-expiration phase. A simple removal of the inconsistent phases from the summed image would then provide a corrected image. The statistical noise in the image would increase with this approach, but the image quality may still be sufficient in many cases, particularly with $\mathrm{NH}_{3}$, for which the counts acquired in the image are much higher than with ${ }^{82} \mathrm{Rb}$.

In this study, we found that the prevalence of respiratory motion $\geq 8 \mathrm{~mm}$ was $7.5 \%$. Of these cases, $39 \%$ (or $3 \%$ of the total number of cases) had significant differences between inspiration and expiration, indicating respiration-induced errors in the images. Although these errors may seem low, compared with other studies $(1,2,25)$, these are the errors present after alignment of the CT scan with the mean (ungated) PET image and so reflect only the errors introduced by the movement of the heart with breathing about its average location. The clinical significance of these errors has not been evaluated. Summation of the respiratory phases would dilute the impact of the differences between inspiration and expiration. In this study, the inspiration and expiration images each contained $25 \%$ of the total data. A significant (3 SDs) difference corresponds to a segmental difference of approximately $15 \%$ or more but only for a quarter of the data in a summed image. The $50 \%$ of the data from mid cycle would also potentially contain differences from expiration, and these would be less than that for inspiration. Thus, the difference between an ungated PET image and the summation of the respiratory phases of the gated PET study with phase-aligned correction could be expected to be on the order of $5 \%$. An example summed image, corresponding to the case shown in Figure 4, is given in Figure 5, along with the difference polar map-which shows segmental errors up to $5.2 \%$. This difference is of a magnitude similar to the variability found by others for cardiac PET flow imaging $(26,27)$. This level of error, though small, still has the potential to degrade the accuracy of PET, considered the gold standard for perfusion imaging, and may alter the severity or extent of disease. Further evaluation of the clinical significance of these errors is required.
Evaluation of the cases with motion $>6 \mathrm{~mm}$ identified another 18 cases with significant error. Inclusion of these cases would raise the prevalence to $9 \%$ of the total cases. However, the focus of this study is on those cases with motion $\geq 8 \mathrm{~mm}$, because the cases with the most motion are those most likely to have errors of clinical relevance.

Additionally, we observe no statistical difference between $\mathrm{NH}_{3}$ and ${ }^{82} \mathrm{Rb}$ imaging with respect to the magnitude of respiratory motion and the prevalence of motion artifacts. The prevalence of respiratory motion artifacts in cases with motion $\geq 8 \mathrm{~mm}$ was the same for both tracers: 5 of 14 for ${ }^{82} \mathrm{Rb}$ and 4 of 9 for $\mathrm{NH}_{3}$. Of the studies with motion $>6 \mathrm{~mm}, 6$ of forty ${ }^{82} \mathrm{Rb}$ and 12 of $33 \mathrm{NH}_{3}$ studies had significant differences between inspiration and expiration when compared with the corresponding no-motion database. This apparent difference in prevalence is likely caused by the greater variability in the ${ }^{82} \mathrm{Rb}$ database (an average of $5.6 \%$ vs. $4.2 \%$ for $\mathrm{NH}_{3}$ ), which is in turn due to the increased noise of the ${ }^{82} \mathrm{Rb}$ images.

\section{Resting Versus Stressed Motion}

We see a small, statistically significant difference between the axial motion measured at rest and stress imaging. This difference is, on average, only $0.3 \mathrm{~mm}$ and would not likely be clinically relevant. However, if we consider just those studies with the largest amounts of motion, there are approximately twice as many stress studies as there are rest. For axial motion $\geq 8 \mathrm{~mm}$, the ratio of stress to rest studies is $9: 5$, and for displacement $\geq 8 \mathrm{~mm}$, the ratio is $11: 6$, suggesting that respiratory motion may be a slightly larger problem for stress cardiac imaging than it is for rest. If correct, a possible cause may be an increase in motion in response to the pharmacologic stress and the fact that acquisition of the PET data is immediately after stress. Because the number of patients in this subgroup is small, further study would be needed to confirm this effect.

There is a correlation between motion at rest and stress $(r=0.6)$. This correlation might be used to help guide the use of respiratory compensation for the second set of (stress) images. The negative predictive value of $\leq 4 \mathrm{~mm}$ of resting motion for stress motion $>6 \mathrm{~mm}$ is $96 \%$. Thus, a rest acquisition showing little motion implies that it is not necessary to apply additional respiratory compensation algorithms at stress, beyond that of registering the CT to 
the static (ungated) PET images. In $45 \%$ of the paired studies, the resting motion was $\leq 4 \mathrm{~mm}$, and so this approach has the potential to greatly reduce the workload on staff for implementing respiratory-motion correction. Even if a 4D-CT technique is being used, avoiding the use of 4D-CT for the stress portion of the study would reduce the dose associated with CT by $33 \%(\sim 1 \mathrm{mSv})$.

\section{Study Limitations}

CT and PET data were aligned manually for this study, and the registration was qualitatively assessed. Other groups have been investigating automated means of registering the CT attenuation map (3), but such software was not available to us on our scanner. These techniques should decrease the time required to perform the alignment, decrease inter- and intraoperator variability, reduce the likelihood of operator error, and potentially provide a more accurate means of aligning the images. Even with automated algorithms, though, a potential error remains. Registering images by matching those that may contain differences because of respiratory motion, as was done in this study, may degrade the accuracy of the registration, particularly for the studies with large amounts of motion, because these images will contain the greatest differences. Further refinement of the registration could be achieved by repeating the registration procedure on the phase-aligned reconstructed PET images.

Gated images were reconstructed using filtered backprojection to stay in accordance with our current clinical practice. Several scans were excluded from this study because of low total counts resulting in poor image quality. Iterative reconstruction-in particular, techniques that use a 4D approach to reconstruction $(5,12,28,29)$ - might provide a better means of reconstruction in these cases. Iterative reconstruction comes, however, at the cost of increased processing time and so would need to be balanced against the processor speed available and clinical workloads.

\section{CONCLUSION}

After registration of end-expiration CT images to static (ungated) PET perfusion images, 39\% of those scans with respiratory motion $\geq 8 \mathrm{~mm}$, representing $3 \%$ of the total scans, still showed residual artifacts. Manual phaseby-phase alignment of an end-expiration 3D CT scan with a respiration-gated PET image reduced respiration-related differences between inspiration and expiration in $89 \%$ of these cases. Phase-aligned CT AC has no additional patient radiation exposure and may improve the specificity of PET perfusion imaging.

\section{ACKNOWLEDGMENTS}

We thank Matt Raegele, May Aung, Kim Gardner, Ann Guo, and the rest of the staff at the National Cardiac PET Centre for their assistance on this project. This research was supported by grant MOP81234 from the Canadian Institutes of Health Research, grant CFI-LEF \#11306 from the Canadian Foundation for Innovation, and program assistance from the Heart and Stroke Foundation of Ontario (PRG6242). Terrence D. Ruddy holds the Vered Chair in Cardiology. Rob S. Beanlands is a Career Investigator for the Heart and Stroke Foundation of Ontario.

\section{REFERENCES}

1. Gould KL, Pan T, Loghin C, Johnson NP, Guha A, Sdringola S. Frequent diagnostic errors in cardiac PET/CT due to misregistration of CT attenuation and emission PET images: a definitive analysis of causes, consequences, and corrections. J Nucl Med. 2007;48:1112-1121.

2. Martinez-Möller A, Souvatzoglou M, Navab N, Schwaiger M, Nekolla S. Artifacts from misaligned CT in cardiac perfusion PET/CT studies: frequency, effects, and potential solutions. J Nucl Med. 2007;48:188-193.

3. Khurshid K, McGough RJ, Berger K. Automated cardiac motion compensation in PET/CT for accurate reconstruction of pet myocardial perfusion images. Phys Med Biol. 2008;53:5705-5718.

4. Souvatzoglou M, Bengel F, Busch R, et al. Attenuation correction in cardiac PET/CT with three different CT protocols: a comparison with conventional PET. Eur J Nucl Med Mol Imaging. 2007;34:1991-2000.

5. Dawood M, Büther F, Jiang X, Schäfers K. Respiratory motion correction in 3-D PET data with advanced optical flow algorithms. IEEE Trans Med Imaging. 2008;27:1164-1175.

6. Fayad H, Lamare F, Bettinardi V, Roux C, Visvikis D. Respiratory synchronized ct image generation from 4D PET acquisitions. 2008 IEEE Nucl Sci Symp Conf Rec. 2008;3:5488-5492.

7. Alessio A, Kohlmyer S, Branch K, Chen G, Caldwell J, Kinahan P. Cine CT for attenuation correction in cardiac PET/CT. J Nucl Med. 2007;48: 794-801.

8. Nye J, Hamill J, Tudorascu D, Carew J, Esteves F, Votaw J. Comparison of low-pitch and respiratory-averaged CT protocols for attenuation correction of cardiac PET studies. Med Phys. 2009;36:1618-1623.

9. Cook R, Carnes G, Lee T, Wells R. Respiratory-averaged CT for attenuation correction in canine cardiac PET/CT. J Nucl Med. 2007;48:811-818.

10. Nagel C, Bosmans G, Dekker A, et al. Phased attenuation correction in respiration correlated computed tomography/positron emitted tomography. Med Phys. 2006;33:1840-1847.

11. Pönisch F, Richter C, Just U, Enghardt W. Attenuation correction of four dimensional (4D) PET using phase-correlated 4D-computed tomography. Phys Med Biol. 2008;53:N259-N268.

12. Lamare F, Carbayo ML, Cresson T, et al. List-mode based reconstruction for respiratory motion correction in PET using non-rigid body transformations. Phys Med Biol. 2007;52:5187-5204.

13. Pan T, Mawlawi O, Nehmeh SA, et al. Attenuation correction of PET images with respiration-averaged CT images in PET/CT. J Nucl Med. 2005;46: 1481-1487.

14. Schwaiger M, Ziegler S, Nekolla S. PET/CT: Challenge for nuclear cardiology. J Nucl Med. 2005;46:1664-1678.

15. Machac J, Bacharach S, Bateman T, et al. Positron emission tomography myocardial perfusion and glucose metabolism imaging. J Nucl Cardiol. 2006;13: e121-e151.

16. Park SJ, Ionascu D, Killoran J, et al. Evaluation of the combined effects of target size, respiratory motion and background activity on 3D and 4D PET/CT images. Phys Med Biol. 2008;53:3661-3679.

17. Dawood M, Büther F, Stegger L, et al. Optimal number of respiratory gates in positron emission tomography: A cardiac patient study. Med Phys. 2009;36: 1775-1784.

18. McLeish K, Hill D, Atkinson D, Blackall J, Razavi R. A study of the motion and deformation of the heart due to respiration. IEEE Trans Med Imaging. 2002;21: 1142-1150.

19. McQuaid S, Hutton B. Sources of attenuation-correction artefacts in cardiac PET/CT and SPECT/CT. Eur J Nucl Med Mol Imaging. 2008;35: 1117-1123.

20. Feng B, Bruyant PP, Pretorius PH, et al. Estimation of the rigid-body motion from three-dimensional images using a generalized center-of-mass points approach. IEEE Trans Nucl Sci. 2006;53:2712-2718.

21. LeMeunier L, Slomka PJ, Fermin J, et al. Motion frozen of dual gated (cardiac and respiratory) PET images [abstract]. J Nucl Med. 2009;50(suppl 2):294P.

22. LeMeunier L, Maass-Moreno R, Carrasquillo JA, Dieckmann W, Bacharach SL. PET/CT imaging: effect of respiratory motion on apparent myocardial uptake. J Nucl Cardiol. 2006;13:821-830. 
23. Livieratos L, Rajappan K, Stegger L, Schafers K, Bailey D, Camici P. Respiratory gating of cardiac PET data in list-mode acquisition. Eur J Nucl Med Mol Imaging. 2006;33:584-588.

24. McQuaid S, Lambrou T, Hutton B. Statistical shape modeling of the diaphragm for application to Rb-82 cardiac PET-CT studies. IEEE Nucl Sci Symp Conf Rec. 2008;3:3651-3655.

25. Lautamäki R, Brown TLY, Merrill J, Bengel FM. CT-based attenuation correction in ${ }^{82} \mathrm{Rb}$-myocardial perfusion PET-CT: incidence of misalignment and effect on regional tracer distribution. Eur J Nucl Med Mol Imaging. 2008;35:305-310.

26. Chareonthaitawee P, Christenson SD, Anderson JL, et al. Reproducibility of measurements of regional myocardial blood flow in a model of coronary artery disease: comparison of $\mathrm{H}_{2}{ }^{15} \mathrm{O}$ and ${ }^{13} \mathrm{NH}_{3}$ PET techniques. J Nucl Med. 2006;47: 1193-1201.

27. Manabe O, Yoshinaga K, Katoh C, Naya M, deKemp RA, Nagara Tamaki N. Repeatability of rest and hyperemic myocardial blood flow measurements with ${ }^{82} \mathrm{Rb}$ dynamic PET. J Nucl Med. 2009;50:68-71.

28. Reyes M, Malandain G, Koulibaly PM, González-Ballester MA, Darcourt J. Model-based respiratory motion compensation for emission tomography image reconstruction. Phys Med Biol. 2007;52:3579-3600.

29. Gravier E, Yang Y, King MA, Jin M. Fully 4D motion-compensated reconstruction of cardiac SPECT images. Phys Med Biol. 2006;51:46034619. 